\title{
SPHERICAL CURVES THAT BOUND IMMERSED DISCS
}

\author{
GEORGE K. FRANCIS
}

\begin{abstract}
Let $f$ be an immersion of the oriented circle in the oriented sphere. Let the image lie in general position and have tangent winding number $\tau$ with respect to some point $\infty$ in its complement. The extensions of $f$ to an orientation preserving immersion of the disc are classified up to topological equivalence by the $\left(0, \frac{1}{2}(1-\tau)\right)$-assemblages induced by a star of rays from $\infty$ to the complementary components of the curve. Applications to the classification problem of stable maps between closed surfaces are also discussed.
\end{abstract}

Introduction. Let $M$ and $W$ be smooth, connected, oriented surfaces, $M$ compact with boundary $\partial M$, and $W$ without boundary. Schoenflies' theorem leads to the natural question of when an immersion $f$ of $\partial M$ in $W$ determines one or more extensions to an immersion of $M$ in $W$. The Stoillow-Whyburn theory of light open maps [18] suggests that a more interesting candidate for extending $f$ would be the following. To be admissible, the extension $F$ shall be an orientation preserving immersion at all but a finite set $B_{F}$ of singularities. At these branch points the map has the topological type of the complex wrapping function $w=z^{m}, m>1$; The multiplicity of the branch point is $\mu_{F}(z)=m-1$. Moreover, the singular values shall not lie on the image of the boundary of $M$.

Unless the image curves $[f]$ lie in general position, that is, $f$ is a normal immersion of $\partial M$ [14], [16], bounding properties may be discontinuous under small deformations. For example, the curves $w_{t}=\cos \theta+$ $i\left(\cos ^{2} \theta+t\right) \sin \theta$ bound plane embedded discs for small positive $t$, but not for $t$ negative. On the other hand, normally immersed circles are combinatorially classifiable by the Titus intersection sequence $[15, \mathrm{p} .49]$.

The Picard-Loewner problem [15, p. 47] asks for a complete combinatorial classification of admissible extension $F$ to $M$ of a normal immersion $f$ of $\partial M$ in terms of the topological invariants of $[f], M$ and $W$. The problem is greatly simplified if $f$ has a spherical target. Let $M$ have genus $\gamma$ and $\partial M$ be the union of $\delta+1$ circles. Set $\mu=\sum \mu_{F}(z), z \in B_{F^{*}}$. Choose a point-at-infinity in the complement of $[f]$ and identify the punctured sphere with the oriented plane. Set $\beta=\sum\left(\mu_{F}(z)+1\right), z \in F^{-1}(\infty)$. Let $\tau$ be

Received by the editors October 26, 1972 and, in revised form, January 30, 1973.

AMS (MOS) subject classifications (1970). Primary 30A48, 57D40; Secondary 55A10, 55G35, 57A70.

(c) American Mathematical Society 1973 
the sum of the tangent winding numbers of the immersed circles belonging to $[f]$ with respect to $\infty$. The necessary condition: $\mu+1=2 \beta+2 \gamma+\delta+\tau$ follows from the work of Morse [12, p. 67]. Conditions for $f$ to have some admissible extension of type $(\mu, \beta, \gamma, \delta)=(\mu, 0,0,0)$ were first given by Titus [15]. His approach was extended by Marx [8], [9] to the cases $(\mu, 0,0,1)$ and $(\mu, 0,1,0)$ and by Marx and Verhey [11] to the $(\mu, \beta, 0,0)$ case. The extensions of a given curve need not, however, be topologically unique. Milnor's curve [13, p. 11] has two $(0,0,0,0)$-extensions. Two admissible extensions are equivalent if they differ by an orientation preserving homeomorphism of $M$. Complete combinatorial descriptions of the equivalence classes of extensions $F$ with these invariants shall be called $(\mu, \beta, \gamma, \delta)$-enumerations. Blank invented the first $(0,0,0,0)$-enumeration [2], [13]. A brief description and application of his idea will be found in [5]. A homotopy approach to the $(\mu, 0,0,0)$-problem was treated in [6]. Marx refined Blank's procedure to obtain a $(\mu, 0,0,0)$-enumeration [10]. Bailey treats the $(0,0, \gamma, 0)$-case in [1]. A $(0,0,0, \delta)$-enumeration was sketched by Blank [3]. This paper presents a $(0, \beta, 0,0)$-enumeration. Some applications are sketched at the end of this paper. The general $(\mu, \beta, \gamma, \delta)$-case will be treated in a future work [7].

Assemblages. We now take $M$ to be the disc $D, \partial M$ its boundary circle $S, W$ the sphere $E \cup\{\infty\}$, and abbreviate $(\mu, \beta, 0,0)$ to $(\mu, \beta)$. From each finite complementary component of $[f]$ draw at least one ray (embedded arc) $\alpha_{i}$ to $\infty$ (so oriented), such that the images $\left[\alpha_{i}\right], 1 \leqq i \leqq r$, intersect only at their common terminus and lie in general position with respect to $[f]$. The rays are indexed clockwise in $E$. Let $a_{i 0}$ be the initial point and $a_{i j}, 1 \leqq j \leqq n(i)$, be the points where $\left[\alpha_{i}\right]$ crosses $[f]$, indexed by their position along the ray. The sign $s= \pm 1$, of the crossing $a_{i j}^{s}$ shall be +1 if $[f]$ crosses from the right to the left side of $\left[\alpha_{i}\right]$, and -1 otherwise. The preimages $x_{i j}=f^{-1}\left(a_{i j}\right)$ comprise the set $X(f, \alpha) \subset S$. Two subsets of $S$ are said to link if each occupies more than one complementary component of the other. A partition of $X$ into mutually nonlinking disjoint subsets will be called a properly nested partition. A singleton has form $\left\{x_{i j}^{+1}\right\}$. A subset of the form $P=\left\{x_{i j}^{-1}, x_{i k}^{+1}\right\}, j<k$, is called a pair. A branch is a subset $B=\left\{x_{i h}^{+1}, x_{i j}^{+1}, \cdots, x_{i k}^{+1}\right\}$, with multiplicity $m(B)=-1+\operatorname{card}(B)$. A subset $C=\left\{x_{i(1) h}^{-1}, x_{i(2) j}^{-1}, \cdots, x_{i(u) k}^{-1}\right\}$, occurring in that circular order on $S$, for which $i(1)<i(2)<\cdots<i(u)$, shall be called a wheel. A properly nested partition $\mathscr{A}$ of $X$ into singletons, pairs, branches and wheels shall be called an $(m, n)$-assemblage, where $m=\sum m(B)$ and $n$ is the number of wheels in $\mathscr{A}$.

THEOREM. Let $f$ be a normal immersion of the oriented circle in the oriented sphere. Let $\mathfrak{F}$ be the set of equivalence classes of admissible extensions 
of $f$ to an orientation preserving immersion of the disc in the sphere. Let $\alpha$ be a raying for $f$ and $\tau$ the tangent winding number of $f$ with respect to the $\infty$-point of $\alpha$. Then $\mathcal{F}$ is in one-to-one correspondence with the set of $(0, \beta)$ assemblages on $X(f, \alpha)$ for which $2 \beta+\tau=1$.

REMARK. The absence of wheels in a $(0,0)$-assemblage implies that all negative crossings are paired, and being properly nested, the pairs form what Blank called a grouping. He did not require the pairs to be ordered by the ray orientation. Using special rayings, he could derive this fact from the existence of a grouping. His rayings are nonredundant (exactly one ray issues from each bounded complementary component of $[f])$ and reduced (every set of the form $\left\{x_{i j}^{-1}, x_{i k}^{+1}\right\}$ links its $X$-complement). For an algebraic interpretation of a nonredundant reduced $(0,0)$ assemblage see [13, p. 7] or [5, p. 378]. For a way of reducing $\alpha$ see [13, p. 15] or [5, p. 379]. Using pairs ordered as above, Marx can construct induction arguments based on the number of pairs in a grouping [10]. Since we require more structure for an assemblage we can dispense with the requirement that the raying be reduced. While redundancy plays a role in enumerating branch points, it is of no consequence here. In proving our theorem we shall use two of Marx's results for starting inductions on $\beta$. The second of these was also obtained by Blank under his stronger assumptions on the raying and his weaker assumptions on the assemblage.

Modifying $(f, \alpha)$. Let $\rho$ denote the metric on $S$ and also the induced Hausdorff metric on compact subsets of $S$. Thus,

$$
\rho\left(K, K^{\prime}\right)=\min \left\{V \geqq 0: K \subset V K^{\prime} \text { and } K^{\prime} \subset V K\right\},
$$

where $K, K^{\prime}$ compact and $V K=\left\{x \in S: \min _{y \in K} \rho(x, y) \leqq V\right\}$. Let $[g]$ be an embedding of $S$ in $E$ completely surrounding [ $f]$ and so that each ray of $\alpha$ cuts $[g]$ once transversally. Label these new, positive crossings $a_{i}^{+1}$. Let ${ }_{j k} \alpha_{i}$ denote the arc segment of $\alpha_{i}$ oriented from $a_{i j}$ to $a_{i k}$. Suppose there is a negative crossing in the $h$ place of the last ray, $a_{r h}^{-1}$. Use a sufficiently narrow tubular neighborhood of $\left[h_{h} \alpha_{r}\right]$ to construct the connected sum $f^{\prime}$ of $f$ and $g$ along $\alpha$. See Figure 1. (In the notation of [11, p. 42] $f^{\prime}$ is a normalization of $f$ aug $1, g, *_{h} \alpha_{r}, a_{r h}, a_{r}$. ) Parametrize $f^{\prime}$ to coincide with $f$ on the oriented arc $\left[x_{r h}+4 V, x_{r h}+V\right]$ on $S$, where $9 V=\min \left\{\rho\left(x^{\prime}, x^{\prime \prime}\right): x^{\prime} \neq\right.$ $x^{\prime \prime}$ in $X$ \}. Then $f^{\prime}$ continues from $f\left(x_{r h}+V\right)$ along an arc parallel to $\left[{ }_{h} * \alpha_{r}\right]$ until it reaches $[g]$ at $g\left(x_{r^{*}}-V\right)$ say. The new curve $f^{\prime}$ continues from there along $[g]$, but parametrized on $\left[x_{r h}+2 V, x_{r h}+3 V\right]$, whence it returns to $f\left(x_{r h}+4 V\right)$ along an arc parallel to $\left[{ }_{* h} \alpha_{r}\right]$. Let $A_{r h}$ denote the topological disc on the sphere containing $\infty$ and bordered by $[f]$ and $\left[f^{\prime}\right]$ both restricted to $\left[x_{r h}+V, x_{r h}+4 V\right]$. The new self-intersections of $\left[f^{\prime}\right]$ shall be assumed to occur at $f\left(x_{r j}^{s}-s V\right)$ and $f\left(x_{r j}^{s}-4 s V\right), j>h$. The newly formed 


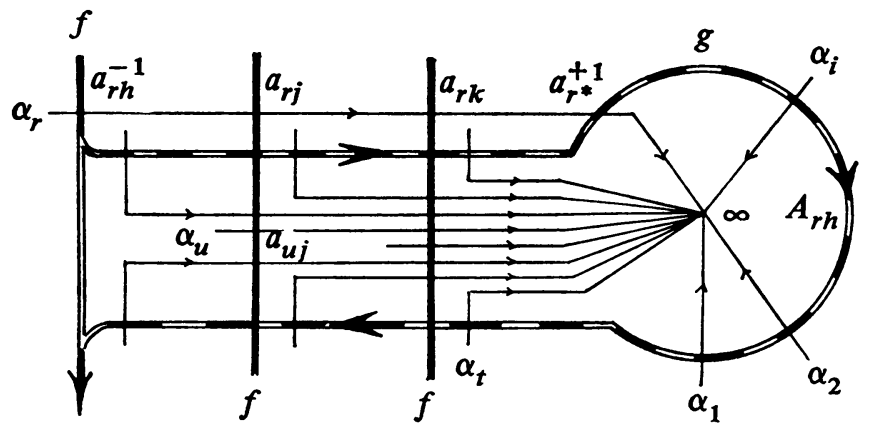

Figure 1

complementary components of $\left[f^{\prime}\right]$ are rayed as indicated in the figure by $t=3(n(r)-h)+2$ further rays $\alpha_{u}, u>r$. In contrast to the convention for $\alpha$, we label the new crossings $a_{u j}$ with the index $j$ belonging to the nearest point in $X$, which is $x_{r j}$. Thus $X \subset X^{\prime}$ and $X^{\prime} \subset 4 V X$. More precisely, the new crossings are $x_{u j}^{s} \in 4 V\left\{x_{r j}^{s}\right\}$ and $\left\{x_{i^{*}}^{+1}, x_{u h}^{+1}\right\} \subset 4 V\left\{x_{r h}^{-1}\right\}, h \leqq j \leqq n(r)$, $1 \leqq i \leqq r<u \leqq t$.

Proposition 1. Given an $(m, \beta)$-assemblage $\mathscr{A}$ on $X(f, \alpha)$ with $\beta \geqq 1, a$ wheel $C_{0}$ together with one of its elements induces an $(m, \beta-1)$-assemblage $\mathscr{A}^{\prime}$ on $X^{\prime}\left(f^{\prime}, \alpha^{\prime}\right)$. The inclusion $X \subset X^{\prime}$ determines an injection $\mathscr{A}-\left\{C_{0}\right\} \rightarrow \mathscr{A}^{\prime}$, $\tau^{\prime}=\tau+2$, and if $f^{\prime}$ has a $(0, \beta-1)$-extension, then $f$ has a $(0, \beta)$-extension.

Proof. For every properly nested partition $X=\bigcup X_{j}, \bigcup 4 V X_{j}$ is a properly nested partition of $4 V X$. Now assume $x_{r h}^{-1} \in C_{0}$. Every member $x_{i j}^{-1}$ of this wheel we now pair with $x_{i *}^{+1}$ of $X^{\prime}$. For every other new negative crossing $x_{u j}^{-1} \in 4 V\left\{x_{r j}^{-1}\right\}, j>h$, and $x_{r j}^{-1}$ either belongs to an old pair or wheel in $\mathscr{A}$. In the former case, $\mathscr{A}$ contains $P=\left\{x_{r j}^{-1}, x_{r k}^{+1}\right\}$, some $k>j$. Hence we pair $x_{u j}^{-1}$ with $x_{u k}^{+1}$ in $X^{\prime}$. In the latter case we enlarge the wheel containing $x_{r j}^{-1}$ by $x_{u j}^{-1}$. It is clear from the construction that the ordering condition on the new wheels is satisfied. To see that no linking occurs in this partition of $X^{\prime}$ we need only check that the pairs $\left\{x_{i j}^{-1}, x_{i^{*}}^{+1}\right\}, x_{i j}^{-1} \in C_{0}$, are mutually nonlinking and that $\left\{x_{u j}^{-1}, x_{u k}^{+1}\right\}$ does not link $\left\{x_{r j}^{-1}, x_{r k}^{+1}\right\}$. In the first case, by assumption the circular order of the $x_{i j}^{-1}$ is increasing in $i$, and by construction, it is decreasing on $i$ for the $x_{i *}^{+1}$. For the second case, we note that the circular order is $x_{r j}^{-1}, x_{u j}^{-1}, x_{u k}^{+1}, x_{r k}^{+1}$. The remaining new positive crossings are assigned to singletons. Thus, except for the breaking up of the wheel $C_{0}$ into pairs, and possible enlarging of old wheels, $\mathscr{A}-\left\{C_{0}\right\}$ is a "subset" of $\mathscr{A}^{\prime}$. Hence $\mathscr{A}^{\prime}$ has the same branching number but one fewer wheel than $\mathscr{A}$. Clearly, $f^{\prime}$ has two further tangential rotations than $f$. Finally, if $f^{\prime}$ bounds a spherically immersed disc, so does $f$. In effect, we attach the embedded disc $A_{r h}$ to the immersed disc bounded 
by $\left[f^{\prime}\right]$ along the simple arc in its boundary given by $f^{\prime}\left[X_{r h}+V, x_{r h}+4 V\right]$. In the language of $\left[4\right.$, p. 277] $f$ is a positive detour of $f^{\prime}$ and we add on the disc as in [4, p. 278].

Proposition 2. If $f$ has a $(0, \beta)$-extension $F$ then for any raying $\alpha, F$ determines a unique $(0, \beta)$-assemblage $\mathscr{A}_{F}$.

Proof. Since $F$ is an immersion we use "analytic continuation" to lift ${ }_{j \infty} \alpha_{i}$ from $x_{i j}^{-1} \in X \subset S=\partial D$ to $D$. Let $z \in$ Clos $D$ be the terminal point of the maximal lift. If $z \in \partial D$ then $z=x_{i k}^{+1}$ for some $k>j$. Assign the pair $P=\left\{x_{i j}^{-1}, x_{i k}^{+1}\right\}$; call the lifted arc the chord $\chi_{P}$. Note that $F$ is an embedding in a neighborhood of $\left[\chi_{P}\right]$ with ${ }_{j k} \alpha_{i}=F \circ \chi_{P}$. If $z \in$ Int $D$ then $F(z)=\infty$ and there are $(r-1)$ further lifts of the remaining rays $\alpha_{k}, k \neq i$ emanating from $z$, ordered clockwise. Following those spokes that terminate on $\partial D$, we assign their terminals to a wheel. The star shaped configuration in Clos $D$ is denoted by $\sigma_{C}$. Again $F$ is an embedding in a neighborhood of $\left[\sigma_{C}\right]$. Since the chords, spokes and the rim $S$ form a planar graph, the corresponding pairs and $n$ wheels are mutually nonlinking. The remaining elements of $X$ are all positive and may be assigned to an arbitrary set of branches and singletons, subject only to the condition that we arrive at some $(m, n)$-assemblage. This is not unique unless $m=0$, in which case the only possible assignment of this remainder is to singletons.

We show that $(m, n)=(0, \beta)$ by induction on $n$. If $n=0, f$ also has a $(\mu, 0)$-extension $F^{\prime}$ with $\mu \geqq m$, as Marx showed in [10, Theorem 6]. Applying Morse's formula to both $F$ and $F^{\prime}$ we have $1-2 \beta=\tau=1+\mu$. Since $\beta$ and $\mu$ are both nonnegative, $0=\beta=\mu=m=n$. Now suppose $n \geqq 1$ and $z$ is the center of the star $\sigma_{C}$. Let $D_{\infty}$ be a sufficiently small disc about $z$ in $D$ which embeds under $F$ into the complement of $[f] ;\left[F \mid \partial D_{\infty}\right]$ shall cut all rays of $\alpha$ once and transversally. Let $g$ be this contour oriented clockwise about $\infty$. Along some spoke, say $\sigma_{r}$ with $h_{\infty} \alpha_{r}=F \circ \sigma_{r}$, we lift to $A^{*}$ the disc $A_{r h}$ constructed in the modification of $(f, \alpha)$ by $g$ described above. Thus $F \mid A^{*}$ embeds $A^{*}$ onto $A_{r h}$ and $f^{\prime}=F \mid \partial D^{\prime}$ where $D^{\prime}=$ $D-\operatorname{Clos} A^{*}$. By Proposition 1 we have the induced $(m, n-1)$-assemblage on $\left(f^{\prime}, \alpha^{\prime}\right)$. Moreover, it is obtained by lifting along the $(0, \beta-1)$-extension $F^{\prime}$ of $f^{\prime}$. So, by induction, $(m, n-1)=(0, \beta-1)$.

Proposition 3. If $(f, \alpha)$ has a $(0, \beta)$-assemblage $\mathscr{A}$ with $\tau=1-2 \beta$, then it has a $(0, \beta)$-extension $F_{. \alpha}$ that induces the same assemblage on $(f, \alpha)$.

Proof. We induct on $\beta$. For $\beta=0, f$ also has a $(\mu, 0)$-extension $F$ that induces the same pairs [10, Theorem 6]. Since $1=\tau=\mu+1, F$ is an immersion. Now suppose $\beta \geqq 1$. By Proposition 1 , the choice of a wheel and spoke induces a $(0, \beta-1)$ assemblage on $\left(f^{\prime}, \alpha^{\prime}\right)$. By induction, $f^{\prime}$ has a $(0, \beta-1)$-extension $F^{\prime}$ inducing the same assemblage on $\left(f^{\prime}, \alpha^{\prime}\right)$. We add 
the disc $A_{r h}$ on to $F^{\prime}$ to obtain $F$, a $(0, \beta)$-extension of $f$. Since $X \subset X^{\prime}, \mathscr{A}_{F}$ is the assemblage we started with.

Proposition 4. Two $(0, \beta)$-extensions of $f$ induce identical $(0, \beta)$ assemblages on $X(f, \alpha)$ if and only if they are equivalent.

Proof. If the two extensions, $F$ and $G$, are equivalent then they coincide on $S$. The equivalence preserves the chords and stars of $F$ and $G$. Hence their pairs and wheels coincide. The converse holds for $\beta=0$ as Marx showed in [10, Theorem 8]. Now suppose $\beta \geqq 1$. Choose $g$ and the tubular neighborhood of ${ }_{n *} \alpha_{r}$ such that the two lifts $A_{F}^{\prime}$ and $A_{G}^{\prime}$ of $A_{r h}$ are both discs in $D$. Their complementary discs $D_{F}^{\prime}=\operatorname{Clos}\left(D-C \cos A_{F}^{\prime}\right)$ and $D_{G}^{\prime}$ are isomorphic under $H^{*}$. Then $G^{\prime} \circ H^{*}$ and $F^{\prime}$ are both extensions of $F \mid \partial D_{F}^{\prime}=f^{\prime}$, where $F^{\prime}=F \mid \operatorname{Clos} D_{F}^{\prime}$ and $G^{\prime}=G \mid$ Clos $D_{G}^{\prime}$. By induction, we have an automorphism $H^{\prime}$ of $D_{F}^{\prime}$ with $G^{\prime} \circ H^{*}=F^{\prime} \circ H^{\prime}$. Now define the equivalence $G=F \circ H$ by setting $H \mid D_{G}^{\prime}=H^{\prime} \circ H^{*-1}$ and $H \mid A_{G}^{\prime}=\left(F \mid A_{F}^{\prime}\right)^{-1}$ 。 $\left(G \mid A_{G}^{\prime}\right)$.

Proof of The Theorem. Associate the equivalence class $\langle F\rangle$ with the uniquely induced $(0, \beta)$-assemblage (Proposition 2$)$ of any representative (Proposition 4). Note that $2 \beta+\tau=1$. By Proposition 3, the association exhausts every such assemblage.

Applications. This $(0, \beta, 0,0$,)-enumeration solves, in effect, the problem [13, p. 33] and [5, p. 383] of classifying immersed discs (with normal boundaries) in any closed 2-manifold. It also contributes to the complete combinatorial classification of the topologically stable maps $G$ between closed surfaces $N$ and $N_{0}$. According to Whitney [17, p. 295] these maps present folds and cusps. That is, the singular set $Z_{G}$ consists of a finite number of disjoint circles in $N$ where $G$ has the topological type of $w=x^{2}+i y$ (fold) or $w=\left(x y-x^{3}\right)+i y$ (cusp). The singular values comprise a finite system of cusped closed curves lying in general position in $N_{0}$. Suppose, for example, that $N$ is the torus and $N_{0}$ is the sphere. Since there can be no immersion of $N$ in $N_{0}$ we may begin with maps having a single

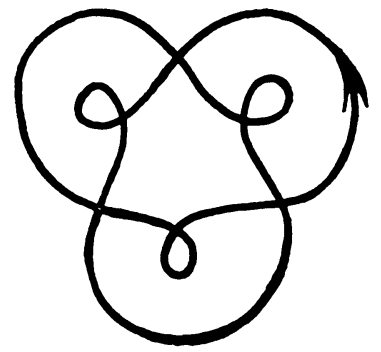

FIGURE 2 
fold. Then $Z_{G}$ is a separating circle on the torus, normally immersed by $G$ in the sphere. If $\tau$ is its tangent winding number, the $\left(0,-\frac{1}{2}(\tau+1), 1,0\right)$ and $\left(0, \frac{1}{2}(1-\tau), 0,0\right)$ enumerations classify these stable maps. For example, the looped trefoil (Figure 2) has a topologically unique extension to a once folded mapping of the torus in the sphere.

ConClusion. The author wishes to thank the sponsors of the 1972 International Colloquium on Topology at Strasbourg for the opportunity of discussing these questions profitably with S. J. Blank and C. J. Titus.

\section{REFERENCES}

1. K. Bailey, Extending closed plane curves to immersions of a disc with $n$ handles, Dissertation, University of Illinois, Urbana, Ill., 1973.

2. S. J. Blank, Extending immersions of the circle, Dissertation, Brandeis University, Waltham, Mass., 1967.

3. - Oral communication, 1972.

4. G. K. Francis, The folded ribbon theorem, A contribution to the study of immersed circles, Trans. Amer. Math. Soc. 141 (1969), 271-303. MR 39 \#4863.

5. - Extensions to the disk of properly nested plane immersions of the circle, Michigan Math. J. 17 (1970), 377-383. MR 44 \#2209.

6. _- Titus' homotopies of normal curves, Proc. Amer. Math. Soc. 30 (1971), 511-518. MR 44\#2233.

7. - Branched and folded parametrizations of the sphere, Bull. Amer. Math. Soc. (to appear).

8. M. L. Marx, Normal curves arising from light open mappings of the annulus, Trans. Amer. Math. Soc. 120 (1965), 46-56. MR 33 \#3278.

9. - Light open mappings on a torus with a disk removed, Michigan Math. J. 15 (1968), 449-456. MR 38 \#2750.

10. - Extensions of normal immersions of $S^{1}$ into $R^{2}$, Trans. Amer. Math. Soc. (to appear).

11. M. L. Marx and R. Verhey, Interior and polynomial extensions of immersed circles, Proc. Amer. Math. Soc. 24 (1970), 41-49. MR 40 \#5879.

12. M. Morse, Topological methods in the theory of functions of a complex variable, Ann. of Math. Studies, no. 15, Princeton Univ. Press, Princeton, N.J., 1947. MR 9, 20.

13. V. Poenaru, Extensions des immersions en codimension 1 (d'apres Samuel Blank), Sem. Bourbaki 1967/68, Exposé 342.

14. C. J. Titus, $A$ theory of normal curves and some applications, Pacific J. Math. 10 (1960), 1083-1096. MR 22 \#5014.

15. - The combinatorial topology of analytic functions on the boundary of a disc, Acta Math. 106 (1961), 45-64. MR 29 \#3652.

16. H. Whitney, On regular closed curves in the plane, Compositio Math. 4 (1937), 276-284.

17. - On singularities of mappings of Euclidean spaces, I. Mappings of the plane into the plane, Ann. of Math. (2) 62 (1955), 347-410. MR 17, 518.

18. G. Whyburn, Topological analysis, 2nd rev. ed., Princeton Math. Series, no. 23, Princeton Univ. Press, Princeton, N.J., 1964. MR 29 \#2758.

Department of Mathematics, Coble Hall, University of Illinois, Urbana, ILLINOIS 61801 